Bull. Mater. Sci., Vol. 9, No. 1, March 1987, pp. 47-54. (C) Printed in India.

\title{
Influence of substrate temperature on the electrical and optical properties of amorphous germanium films
}

\author{
S V CHANDOLE*, UJWALA V HULSURKAR and S S SHAH \\ Department of Physics, Marathwada University, Aurangabad 431004 , India \\ *Department of Physics, Deogiri College, Aurangabad 431 005, India
}

MS received 15 October 1984; revised 28 July 1986

\begin{abstract}
The influence of substrate temperature on electrical and optical properties of the amorphous germanium films deposited under well-defined conditions has been investigated. DC electrical conductivity in the temperature range of $80-573^{\circ} \mathrm{K}$ has been measured. In the low temperature region Mott's $T^{-1 / 4}$ law of conductivity is obeyed. The estimated values of $T_{0}$ and $N$ show significant decrease with change in $T_{s}$ in steps of $50^{\circ} \mathrm{K}$. Similar results are seen in annealed films. The values of activation energy and optical energy increase with $T_{s}$.
\end{abstract}

Keywords. Amorphous germanium; annealing; substrate temperature; density of states; optical energy gap; d.c. electrical conductivity.

\section{Introduction}

Although much work has been carried out on the structural, electrical and optical properties of amorphous germanium (a-Ge), the many aspects towards a basic understanding of a-Ge have still remained a challenging problem. This is due to the strong dependence of properties such as density, structural transformation kinetics, electrical conductivity, thermopower, magneto-resistance, optical absorption and optical constants etc observed on various deposition parameters, subsequent ageing, annealing treatment and adsorption effects.

An amorphous thin film obtained by condensation of vapour atoms onto the substrate consists of various defects in the films like voids, vacancies, divacancies, dangling bonds (unsatisfied bonds) etc and these defects arise due to various preparation conditions during deposition and pre- and post-deposition treatments of the films. Thermally-activated continuous rearrangement is possible in amorphous materials if the amorphous matrix contains micropolycrystalline regions which may occur in various deposited films and if finite adatom mobility exists during the condensation process. Though the existence of the so-called ordered region of $\simeq 14 \AA$ dimensions (Graczyk and Choudhari 1973) hardly supports the microcrystalline nature of a-Ge films, the sharp dependence of several properties of a-Ge films on various deposition parameters (Walley 1968; Bauer and Galeener 1972; Zavetova and Koc 1972; Goebel et al 1973) can be understood only on the assumption of a finite adatom mobility during the condensation process of the film. Therefore, for a satisfactory interpretation of the properties, a clear understanding of the atomic rearrangement during and after deposition of a-Ge film is necessary. This is because any rearrangement in the structure of films causes a change in the number of defects thus providing a variation in the density of states in the mobility gap and therefore in the properties. Studies on the variation of angle of deposition (Chopra and Pandya 1974), annealing (Walley 1968; Brodsky et al 1970; Brodsky and Title 1969; Paesler et al 1974; Paul and Mitra 1973) and oxygenation (Walley 1968; Lecomber et al 
1974) in a-Ge revealed a decrease in the density of states with an increasing angle of incidence, annealing temperature $T_{A}$ and the presence of oxygen during deposition respectively.

We have utilized the substrate temperature as a parameter while studying the d.c. electrical conductivity of vacuum-evaporated a-Ge films in the temperature range $80-573^{\circ} \mathrm{K}$. The optical absorption of samples deposited at various substrate temperatures has also been studied.

\section{Experimental}

\subsection{Sample preparation}

Sample films of a-Ge were prepared by evaporating intrinsic $(99.99 \%$ pure) Ge from molybdenum boat onto the clean glass substrates with predeposited Al contacts held at various substrate temperatures. The six substrate temperatures chosen were in the range of $300-500^{\circ} \mathrm{K}$ in steps of $50^{\circ} \mathrm{K}$. The angle of deposition was $\simeq 80^{\circ}$. The deposition rate was $\simeq 100 \AA / \mathrm{sec}$. All evaporations were made using a conventional vacuum system with an oil diffusion pump (pressure during deposition $10^{-5}$ torr). Film thickness was measured using an interferometric technique. The thickness of the film was $1000-1500 \AA$.

\section{$2.2 D C$ conductivity measurement}

The low temperature d.c. conductivity was measured by transferring sample films after deposition to the cryostat (Chandole and Shah 1981) evaluated at a pressure of $10^{-3}$ torr. The sample conductance was measured at various stable temperatures between room temperature and upto $130^{\circ} \mathrm{K}$. While measuring the conductance, pressure contacts were used and the temperature was recorded using a calibrated copper constantan thermocouple fixed on a glass plate near the sample. High temperature d.c. conductivity was measured from room temperature to $573^{\circ} \mathrm{K}$ in vacuum and $\simeq 10^{-5}$ torr pressure. The sample conductance was measured at different temperatures. While measuring the conductance, pressure contacts were used. Both at low and high temperatures, conductance was measured by determining a voltage drop across a standard resistor using a d.c. micro-voltmeter TFM 12.

If the natural logarithm $(\ln ) \sigma_{\mathrm{dc}}$ vs $T^{-\frac{1}{3}}$ is plotted using a low temperature $\sigma_{\mathrm{dc}}$ data, we get a straight line plot, the slope of which will be $T_{0}^{\frac{1}{4}}$. Thus using experimentally obtained values of $T_{0}$, the values of density of states at $E_{F}, N\left(E_{F}\right)$ were obtained using the relation (Ambegaonkar et al 1971) $T_{0}=16 \alpha^{3} / K N\left(E_{F}\right)$ where $\alpha=10^{7} \mathrm{~cm}^{-1}$ and $k$ is the Boltzmann's constant. Also by plotting $\ln \sigma_{\mathrm{dc}} \mathrm{vs} 1 / T$ for high temperature $\sigma_{\mathrm{dc}}$ data, the values of $E_{\mathrm{act}}$ were determined for various sample films from the slope of the plots. The values of $N\left(E_{F}\right)$ and $E_{\text {act }}$ are shown in table 1. The values of $\ln \sigma_{\mathrm{dc}}$ vs $T^{-\frac{1}{2}}$ and $\ln \sigma_{\mathrm{dc}}$ vs $1 / T$ plots are shown in figures 1 and 2 respectively.

\subsection{Optical absorption}

The optical absorption of the sample films was measured using a double beam spectrometer (Cary 17D) in the near infrared range (8000-20000 $\AA$ ). The refractive index 
Table 1. Electrical and optical data of a-Ge films.

\begin{tabular}{|c|c|c|c|c|}
\hline $\begin{array}{l}\text { Substrate } \\
\text { temperature } \\
T_{s}\left({ }^{\circ} \mathrm{K}\right)\end{array}$ & $\begin{array}{c}\text { Hopping } \\
\text { parameter } \\
T_{0}\left({ }^{\circ} \mathrm{K}\right)\end{array}$ & $\begin{array}{c}\text { Density of } \\
\text { states } \\
N\left(E_{F}\right)\left(\mathrm{eV}^{-1} \mathrm{~cm}^{-3}\right)\end{array}$ & $\begin{array}{c}\text { Activation } \\
\text { energy } \\
E_{\text {act }}(\mathrm{eV})\end{array}$ & $\begin{array}{c}\text { Optical } \\
\text { energy } \\
E_{g}^{\mathrm{op}}(\mathrm{eV})\end{array}$ \\
\hline 300 & $5 \cdot 39 \times 10^{7}$ & $3.44 \times 10^{18}$ & 0.32 & 0.86 \\
\hline 350 & $7.72 \times 10^{7}$ & $2.40 \times 10^{18}$ & $0 \cdot 33$ & $0 \cdot 88$ \\
\hline 400 & $1.60 \times 10^{8}$ & $1.16 \times 10^{18}$ & $0 \cdot 35$ & 0.90 \\
\hline 450 & $1.99 \times 10^{18}$ & $9.33 \times 10^{37}$ & 0.36 & 0.93 \\
\hline 500 & $2.93 \times 10^{8}$ & $6.32 \times 10^{17}$ & 0.45 & 0.96 \\
\hline
\end{tabular}

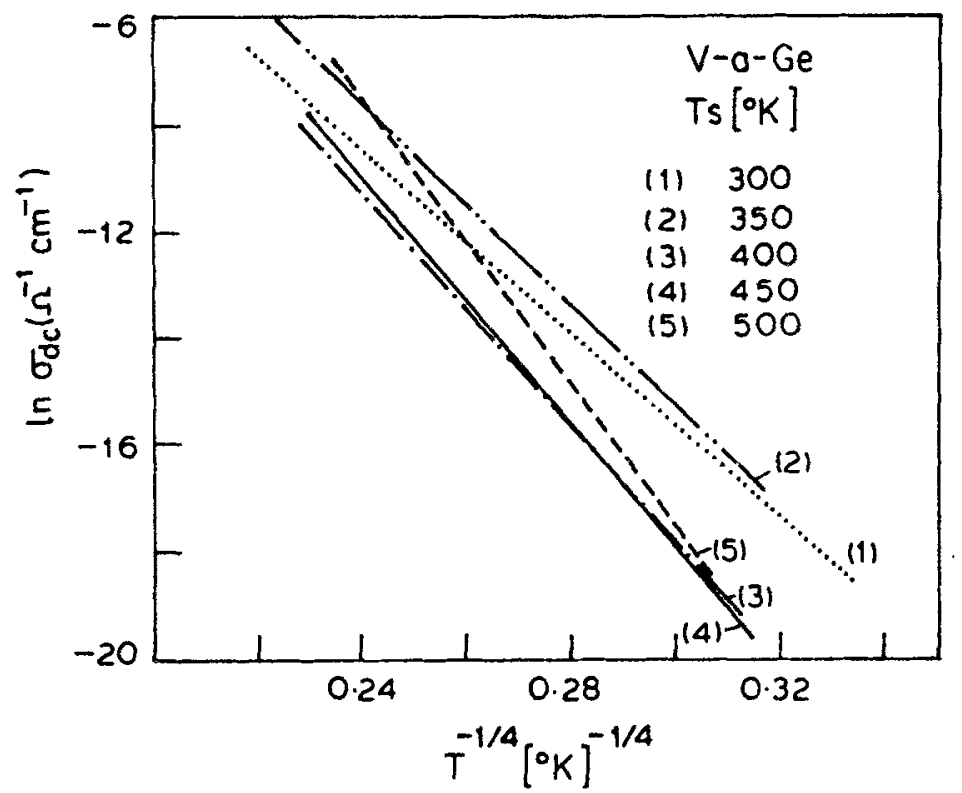

Figure 1. Low temperature measurement data plotted for a-Ge films with different $T_{s}$ values.

(n) of the sample films was determined by identifying the order of interference peak obtained from transmission maximum and transmission minimum (Wales et al 1967). An average value of $n$ was calculated and used for further calculation. Following Brodsky et al (1970) for a film of thickness, the value of transmission $T$ is given by

$$
T=\frac{\left(1-R_{1}\right)\left(1-R_{2}\right)\left(1-R_{3}\right) \exp (-\alpha t)}{1-R_{2} R_{3}\left[1-\left|R_{1} R_{2}+R_{1} R_{3}\left(1-R_{2}\right)^{2}\right| \exp (-\alpha t)\right]},
$$

where $R_{1}$ is the film to air reflection coefficient $=[(n-1) /(n+1)]^{2}, R_{2}$ is the film substrate reflection coefficient $=\left[\left(n-n_{s}\right) /\left(n+n_{s}\right)\right]^{2}, R_{3}$ is the air to substrate reflection coefficient $=\left[\left(1-n_{s}\right) /\left(1+n_{s}\right)\right]^{2}, \alpha$ is the absorption coefficient, $n$, the refractive index of glass and $n_{s}$ is the refractive index of the film. It is found that $R_{3} \ll R_{2}<R_{1}$, so that the above formulae can be approximated to the form

$$
T=\left(1-R_{1}\right)\left(1-R_{2}\right)\left(1-R_{3}\right) \exp (-\alpha t) .
$$




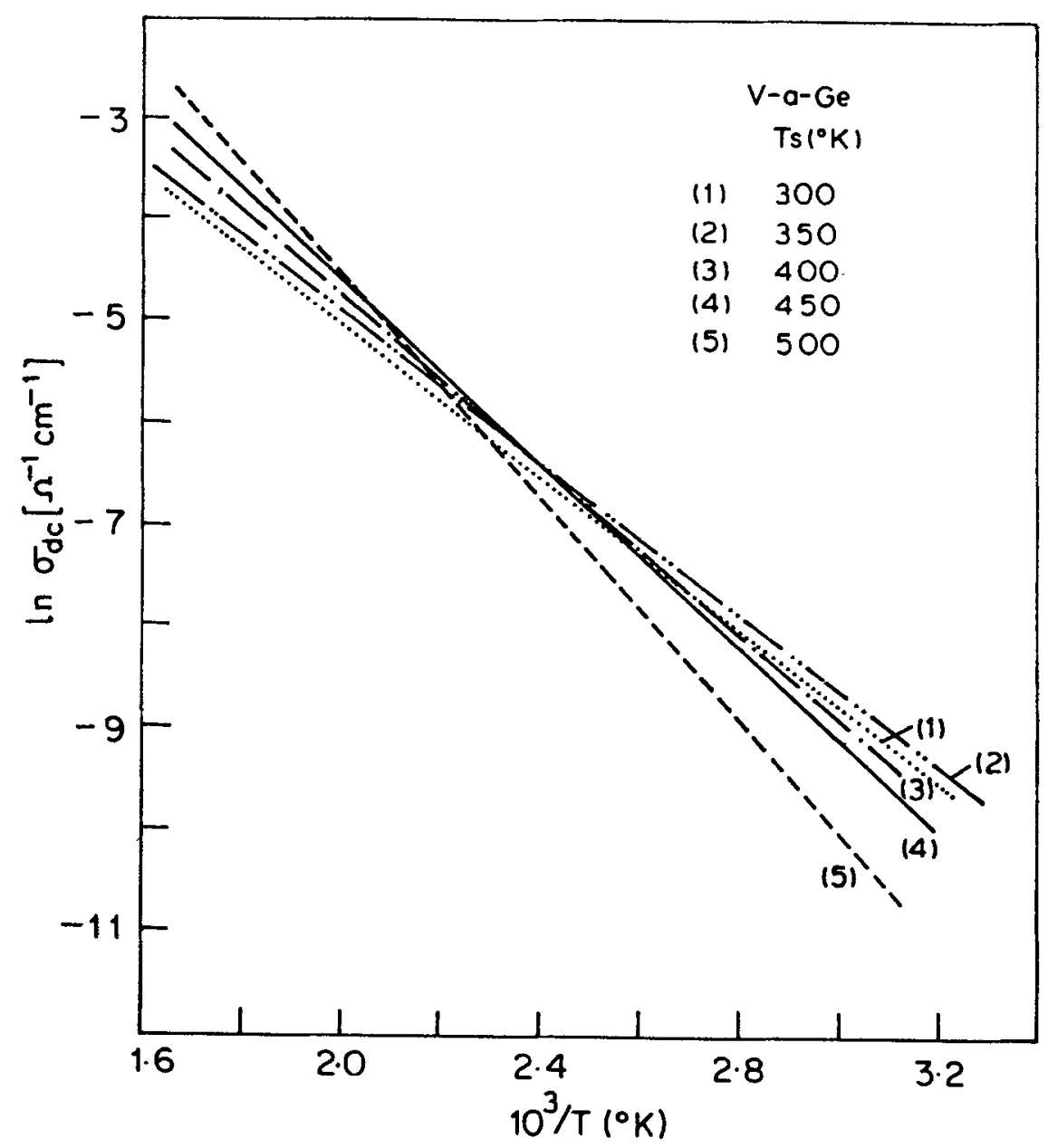

Figure 2. High temperature measurement data plotted for a-Ge films with different $T_{s}$ values.

From the value of $T$ measured experimentally, $\alpha$ can be calculated using the above equation. When absorption is plotted as $(\alpha h v)^{\frac{1}{2}}$ vs $\left(h v-E_{g}^{o p}\right)$, a straight line fit for the above data (particularly at the high absorption region) is observed. Extrapolating this to $\alpha=0$ gives the value of $E_{g}^{\text {op }}$. By measuring $T$ experimentally, the plots of $\alpha$ vs $h v$ and $(\alpha h v)^{\frac{1}{2}}$ vs $h v$ for all samples were obtained to study the absorption edge and to calculate the $E_{g}^{o p}$ respectively. The $E_{g}^{o p}$ data are summarized in table 1 and plots $\alpha$ vs $h v$ and $(\alpha h v)^{\frac{1}{2}}$ vs $h v$ are shown in figures 3 and 4 respectively.

\section{Results and discussion}

The low temperature dependence of d.c. conductivity in a-Ge films deposited at $T_{s}=300^{\circ} \mathrm{K}$ and at elevated $T_{s}$ is shown in figure 1 . Mott's $T^{-\frac{1}{4}}$ law of conductivity is verified in each case. As the resistivity of the films increase with higher $T_{s}$ values $T^{-\frac{1}{4}}$ plots shift towards the lower conductivity region. The values of $T_{0}$ obtained 




Figure 3. Absorption coefficient $(\alpha)$ vs photon energy $(h v)$ plotted for a-Ge films with different $T_{s}$ values.

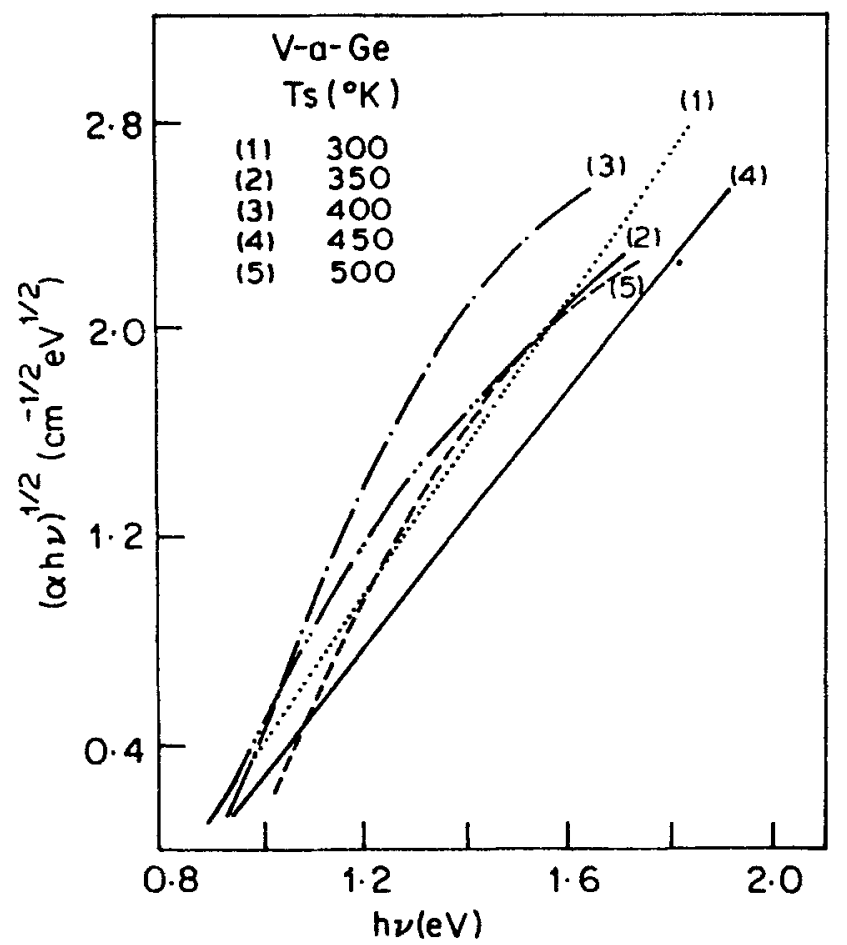

Figure 4. $(x h v)^{\frac{1}{2}}$ vs photon energy $(h v)$ plotted for a-Ge films with different $T_{s}$ values. 
from the $T^{-1}$ plots increase with increasing value of $T_{s}$. Thus $N\left(E_{F}\right)$ is smaller in films deposited at elevated $T_{s}$. This observation agrees with the studies on annealed (Hasegawa et al 1978; Paul and Mitra 1973; Narasimham 1977; Koc et al 1972) and oxygenated a-Ge films (Zavetova and Koc 1972; Lewis et al 1974; Kubler et al 1979). The temperature dependence of the conductivity of a-Ge films deposited at various $T_{s}$ is shown in figure 2 . Although the conductivities of the film with $T_{s}=300^{\circ} \mathrm{K}$ and films with increased $T_{s}$ differ considerably from each other, the general features of the temperature dependence behaviour are found to be similar as the value of $E_{\text {act }}$ decreases rapidly with decreasing temperature and at the same temperature $E_{\text {act }}$ is higher for films with higher $T_{s}$. This effect is similar to that observed in annealed (Pierce and Spicer 1971), oxygenated and obliquely deposited (Chopra and Pandya 1974) a-Ge films. The optical absorption coefficients $\alpha$ of the a-Ge films deposited at $T_{s}=300^{\circ} \mathrm{K}$ near the fundamental absorption edge behave nearly in the same manner as that reported by Theye (1971) with increasing $T_{s}$. The curves $\alpha$ vs $h v$ and $(\alpha h v)^{\frac{1}{2}}$ vs $h v$ (figures 3 and 4 respectively) for a-Ge films shift towards higher energies. The value of $E_{g}^{\text {op }}$ for a-Ge film deposited at $T_{s}=300^{\circ} \mathrm{K}$ is in accordance with that reported by Theye (1971). Similar results are reported for optical absorption studies carried out over annealed (Brodsky et al 1970; Hasegawa and Kitagawa 1978; Koc et al 1972; Theye 1971, 1974; Pandya and Chopra 1976), oxygenated (Mott and Davis 1979) and obliquely deposited a-Ge films (Chopra and Pandya 1974). The shift of fundamental absorption edge towards higher energies and increase in $E_{g}^{\text {op }}$ values with increasing $T_{s}$ is also reported by other workers (Pandya and Chopra 1976; Theye 1970; Connell et al 1973; Donovan and Spicer 1970).

In general, films deposited at high $T_{s}$ are of high density and coordination number but they have a fair density of impurities, voids, vacancies, dangling bonds, etc. In such cases trends of these properties with increasing film density allow the effect of dangling bonds, voids, impurities etc to be separated out from those of matrix itself. This happens at crystallization temperature because there is a gradual modification in the electrical and optical properties of films with increasing $T_{s}$ below crystallization temperature. Density, refractive index, resistivity, activation energy and optical energy gap values tend to increase upto limiting values and this is characteristic of most ideal amorphous state of the matrix. Deposition of films at quite high $T_{s}$ may produce high density films closer to those of the hypothetical pure perfectly coordinated network i.e. pure crystalline films (Connell et al 1973). According to Theye (1971) the ideal amorphous state and complete crystallization state of vacuum evaporated a-Ge films occur at $400^{\circ} \mathrm{C}$ and $500^{\circ} \mathrm{C}$ respectively. These limiting states have characterized $E_{g}^{\text {op }}$ values as $1 \mathrm{eV}$ and $0.8 \mathrm{eV}$ respectively. Our films have preparation conditions similar to that of Theye (1971) and the observed changes in the electrical and optical properties of our sample films below crystallization temperature, can be interpreted as showing progressive transformation of the asdeposited film towards a most ideal amorphous state. Our annealing supports such a modification in the film structure with $T_{s}$ as we obtained a value of $E_{g}^{\mathrm{op}} \simeq 0.99 \mathrm{eV}$ (comparable to $\alpha \simeq 1 \mathrm{eV}$, a characteristic value of $E_{g}^{\text {op }}$ indicating perfect amorphous state) in a-Ge annealed at $T_{A}=300^{\circ} \mathrm{C}$ for $6 \mathrm{hr}$ (Delit and Shah 1983).

The role of $T_{s}$ determining such a transformation in the structure of the film and thereby the properties may be understood with the possible existence of finite adatom mobilities. Though adatom mobility is considered very small, it may be finite in the films deposited with the increased temperature of $T_{s}$ since adatom may get 
more thermal activation energy from the surface of substrate compared to that of the film deposited into room temperature substrates. As a result the rearrangement may be different for different $T_{s}$. The resulting gradual change in the structure of films with $T_{s}$ may be related to the probability that a high energy adatom configuration may transform to a lower energy adatom configuration during deposition of a monolayer (Connell et al 1973).

The fact that density of defect states at $E_{F}$ decreases with increasing $T_{s}$ below recrystallization temperature (table 1) supports the explanation that there is a gradual transformation of the as-deposited films towards a most ideal amorphous state with well-defined specific properties. The increase in $T_{0}, E_{\text {act }}$ and $E_{g}^{\text {op }}$ data with $T_{s}$ (table 1) below crystallization temperature also confirms the above explanation as the data reflect the properties of the increasing amorphous state of the matrix. Similar reports on sputtered a-Ge films (Connell et al 1973) have shown that the main structural changes are due to densification of the structure with the elimination of voids, dangling bonds etc.

Thus, similar to annealing and oblique deposition studies, the trends of electrical and optical properties of vacuum-evaporated a-Ge films reflect the same cycle indicating an evolution of the film structure towards an ideal amorphous state with increasing $T_{s}$ below crystallization temperature $\left(\simeq 300^{\circ} \mathrm{C}\right)$. For higher $T_{s}>300^{\circ} \mathrm{C}$ we may expect the modification of the film structure towards recrystallization which, according to annealing studies, are expected to start at about $T_{A} \geqslant 400^{\circ} \mathrm{C}$. This is obvious since densification of the matrix after an ideal amorphous state will result in recrystallization of the structure. Such changes in the films with elevated $T_{s}$ may be detected by performing structural studies in films with elevated $T_{s}$ as seen in the case of annealed films (Theye 1971).

\section{References}

Ambegaonkar V, Halperin B I and Langer J S 1971 Phys. Rev. B4 2612

Bauer R S and Galeener F L 1972 Solid State Commun. 101171

Brodsky M H and Title R S 1969 Phys. Rev. Lett. 23581

Brodsky M H, Title R S, Weisser K and Pettit G D 1970 Phys. Rev. B1 2632

Chopra K L and Pandya D K 1974 Proc. Fifth Int. Conf. on Amorphous and liquid semiconductor, (ed) J Stuke and W Brenig (London: Taylor and Francis) p 1141

Chandole S V and Shah S S 1981 Indian J. Cryogenics 682

Chandole S V 1983 Ph.D. thesis, Marathwada University, Aurangabad

Connell G A N, Temkin R J and Paul W 1973 Adv. Phys. 22 531, 643

Donovan T M and Spicer W E 1970 Phys. Lett. A32 85

Goebel H, Dettmer K and Kessler F R 1973 Phys. Status Solidi A16 61

Graczyk J F and Chaudhari P 1973 Phys. Status Solidi B58 501

Hasegawa S and Kitagawa M 1978 Solid State Commun. 27855

Hasegawa S, Yazaki S and Shimizu T 1978 Solid State Commun. 26407

Koc S, Zavetova M and Zemek J 1972 Czech. J. Phys. B22 1296

Koc S, Zavetova M and Zemek J 1972 Thin Solid Fims 10165

Kubler L, Jacgle A and Kouimann J J 1979 Phy's. Status Solidi B95 307

Lecomber P G, Loveland R J, Spear W E and Vaughan R A 1974 Amorphous and liquid semiconductors (eds) J Stuke and W Brenig (London: Taylor and Francis) p. 245

Lewis A J, Connell G A N, Paul W., Pawlik R J and Temkin R J 1974 Tetrahedrally bonded amorphous semiconductors (ed) M H Brodsky (New York: American Institute of Physics) p 27

Mott and Davis 1979 Electronic process in non-crystalline materials (Oxford: Clarendon Press) p 333

Narasimham K L 1977 Ph.D. thesis, TIFR, Bombay 
Paesler M A, Agarwal S C, Hudgens S J and Fritzche H 1974 Tetrahedrally bonded amorphous semiconductors (eds) M H Brodsky, S Kirpatric and D Wearie (New York: American Institute of Physics) p 37

Pandya D K and Chopra K L 1976 Phys. Status Solidi. A35 725

Paul D K and Mitra S S 1973 Phys. Rev. Lett. 311000

Pierce D T and Spicer W E 1971 Phys. Rev. Lett. 271217

Theye M L 1970 Opt. Commun. 2329

Theye M L 1971 Mater. Res. Bull. 6103

Theye M L 1974 Amorphous and liquid semiconductors (eds) J Stuke and W Brenig (London: Taylor and Francis) p 479

Wales J, Lovitt G J and Hill R A 1967 Thin Films 1137

Walley P A 1968 Thin Solid Films 2327

Zavetova M and Koc S 1972 Czech. J. Phys. B22 429 